Association for Information Systems

AIS Electronic Library (AISeL)

Wirtschaftsinformatik Proceedings 2005

Wirtschaftsinformatik

February 2005

\title{
Einfach besser? Zur Anwendbarkeit des industriellen Komplexitätsmanagements auf variantenreiche IT-Dienstleistungen
}

Tilo Böhmann

Technische Universität München

Helmut Krcmar

Technische Universität München

Follow this and additional works at: http://aisel.aisnet.org/wi2005

\section{Recommended Citation}

Böhmann, Tilo and Krcmar, Helmut, "Einfach besser? Zur Anwendbarkeit des industriellen Komplexitätsmanagements auf variantenreiche IT-Dienstleistungen" (2005). Wirtschaftsinformatik Proceedings 2005. 24.

http://aisel.aisnet.org/wi2005/24

This material is brought to you by the Wirtschaftsinformatik at AIS Electronic Library (AISeL). It has been accepted for inclusion in Wirtschaftsinformatik Proceedings 2005 by an authorized administrator of AIS Electronic Library (AISeL). For more information, please contact elibrary@aisnet.org. 
In: Ferstl, Otto K, u.a. (Hg) 2005. Wirtschaftsinformatik 2005: eEconomy, eGovernment, eSociety; 7. Internationale Tagung Wirtschaftsinformatik 2005. Heidelberg: Physica-Verlag

ISBN: 3-7908-1574-8

(C) Physica-Verlag Heidelberg 2005 


\title{
Einfach besser? Zur Anwendbarkeit des industriellen Komplexitätsmanagements auf variantenreiche IT-Dienstleistungen ${ }^{1}$
}

\author{
Tilo Böhmann, Helmut Krcmar
}

Technische Universität München

Zusammenfassung: Höhere Flexibilität und geringere Produktionskosten - dieser Herausforderung müssen sich neue Leistungsangebote wie On-Demand-Services im Markt für IT-Dienstleistungen stellen. Die Hoffnung wird dabei auf neue Standardsvorhaben und technische Innovationen gelegt, mit denen die höhere Komplexität dieser Dienstleistungen beherrscht werden soll. Die Erfahrungen des industriellen Komplexitätsmanagements zeigen aber, dass dieser Weg in eine Komplexitätsfalle führt. Vor der Beherrschung der Komplexität muss ihre Reduktion stehen. Daher untersuchen wir, wie sich Maßnahmen zur Komplexitätsreduktion aus der industriellen Fertigung auf variantenreiche IT-Dienstleistungen übertragen lassen. Dazu wird zunächst in das Komplexitätsmanagement eingeführt, dann die Leistungs- und Gestaltungselemente von IT-Dienstleistungen beschrieben und schließlich die Anwendbarkeit von Maßnahmen entlang eines Fallbeispiels überprüft.

Schlüsselworte: IT-Dienstleistungen, On-Demand, Komplexitätsmanagement, Komplexitätsberrschung, Outsourcing

\section{Einführung}

„On-Demand“ ist eines der beherrschenden Themen im Markt für IT-Outsourcing. Darunter werden gewöhnlich auf Abruf verfügbare IT-Dienstleistungen verstanden, die nutzungsabhängig verrechnet werden. Der Nutzen dieser Leistungsangebote soll einerseits in einer Variabilisierung der IT-Kosten und andererseits in einer höheren Flexibilität für die Deckung schwankender oder schwer planbarer Bedarfe an Leistungen der Informationsverarbeitung liegen.

Bisherige Untersuchungen zeigen, dass vor allem Produktionskostenvorteile bei der Entscheidung für das Outsourcing von IV-Leistungen ausschlaggebend sind [AnSt98]. Anbieter können Produktionskostenvorteile gegenüber einer Eigen-

Die Autoren danken Herrn Oliver Häberle für wertvolle Hinweise zu diesem Beitrag. 
erstellung der Outsourcinggeber durch Skaleneffekte, Verbundeffekte und Lernkurveneffekte erzielen [Lamm04, S. 212]. Die hohe Bedeutung von Produktionskostenvorteilen bei der Entscheidung über einen Fremdbezug legt nahe, dass diese Vorteile auch für bedarfsorientiert abrufbare und nutzungsabhängig verrechnete IT-Leistungen vorliegen müssen. Konkret kann dies bedeuten, dass z. B. die kurzfristige Bereitstellung von Leistungen günstiger realisiert werden kann, als wenn ein Nutzer selbst entsprechende flexibel einsetzbare Ressourcen vorhält.

Das stellt die Anbieter vor die Herausforderung, wie sie mit der hohen Vielfalt dieser Leistungsangebote umgehen. Bei unternehmensbezogenen IT-Leistungen verursachen zunächst allgemein die heterogenen funktionalen und nichtfunktionalen Anforderungen sowie die Anforderungen an die Integration in bestehende Systemlandschaften eine hohe Varietät. Die Anpassbarkeit an die Bedarfsverläufe über die Zeit verstärkt diese Varietät bei den neuen Leistungsangeboten, da die Leistungen weder langfristig festgeschrieben werden, noch Veränderungen an den Leistungen im Vertragszeitraum kundenspezifisch implementiert werden.

Um die erhöhte Varietät ohne einen proportionalen Anstieg der Produktionkosten übernehmen zu können, werden die Hoffnungen in der Regel auf neuere technische Entwicklungen gesetzt. Neue Standardisierungsvorhaben, technische Architekturen und Werkzeuge sollen größere Potenziale für die flexible und automatisierte Produktion von IT-Dienstleistungen eröffnen. Dies gilt sowohl für die Kopplung von Anwendungssystemen (z.B. durch Standards für web services) als auch Virtualisierung von IKT-Infrastrukturen (z. B. durch Standards und technische Innovationen zur Flexibilisierung von Rechenzentrumsressourcen).

Die neue Produktionstechnik soll es demnach ermöglichen, an unternehmensspezifische Anforderungen und den tatsächlichen Bedarfsverlauf anpassbare IVLeistungen zu erstellen. Kurz: die Komplexität von sich wandelnden, unternehmensbezogenen IT-Leistungen soll durch verbesserte Technik effizient beherrscht werden. Diese einseitige Fokussierung auf Komplexitätsbeherrschung durch flexiblere Produktionstechnik birgt aber eine Reihe von Risiken:

1. Entwicklungsrisiko: Viele der für die Realisierung unterstellten technischen Neuerungen befinden sich noch im Entwicklungsprozess. Ob und wie die dafür formulierten Visionen auch tatsächlich umgesetzt werden können, ist noch offen.

2. Wettbewerbsrisiko: Je stärker die neue Technik für alle Unternehmen verfügbar ist, desto geringer sind die Wettbewerbsvorteile, die sich aus dem Zugang zur Technik ergeben können [Carr03]. Dieses Risiko wird durch die prominente Rolle von Standardisierungsvorhaben im Rahmen der technischen Entwicklung erhöht. Lediglich das Volumen der erforderlichen Ausstattungsinvestitionen für den Aufbau der notwendigen Infrastrukturen könnte eine (temporäre) Markteintrittsbarriere darstellen. Je stärker aber diese Infrastrukturen durch Aggregation von heterogenen Systemelementen aufgebaut wer- 
den (z.B. bei Grid Computing und Web Services), desto geringer dürfte auch diese Barriere ausfallen.

3. Komplexitätsrisiko: Die Potenziale der Industrialisierung für Kunden und Anbieter der neuen Leistungsangebote liegen in der effizienten Beherrschung der damit verbundenen Varietät begründet. Erfahrungen aus der Industrie weisen deutlich auf die Gefahr einer Komplexitätsfalle hin, die sich aus einer ungefilterten Erhöhung der Varietät in Markt, Leistungsprogramm und Leistungserstellung ergibt.

Diese Risiken deuten darauf hin, dass eine einseitige Fokussierung der Produktionstechnik ein hohes Misserfolgsrisiko bei Entwicklung, Vertrieb und Erbringung abrufbarer und nutzungsorientiert verrechneter IT-Dienstleistungen mit sich bringt.

Für die industrielle Fertigung ist der Umgang der mit einer hohen Varietät verbundenen Komplexität umfangreich untersucht worden. Die Ergebnisse unterstreichen einerseits die Bedeutung der Komplexitätsreduktion und andererseits die Grenzen der Komplexitätsbeherrschung. In diesem Beitrag soll daher untersucht werden, inwieweit sich die Instrumente der Komplexitätsreduktion auch auf anpassbare und abrufbare IT-Dienstleistungen übertragen lassen, um damit das Komplexitätsrisiko ihrer erfolgreichen Implementierung zu verringern.

Der Beitrag befasst sich damit mit der Anbieterseite von IT-Dienstleistungen, die bislang erst vereinzelt im Kontext der Forschung zu IT-Outsourcing und ITDienstleistungen untersucht wurden [LeRo03; Saun02].

Der Beitrag gliedert sich zu Beginn in zwei Stränge. Im ersten Strang beschreiben wir zunächst die Elemente von IT-Dienstleistungen und ihre Abhängigkeiten. Dies legt die Grundlage für ein Verständnis für die Verursacher von Komplexität bei IT-Dienstleistungen. Gleichzeitig wird so deutlich, welche Besonderheiten bei der Anwendung des industriellen Komplexitätsmanagements auf IT-Dienstleistungen berücksichtigt werden müssen. Im zweiten Strang gehen wir anschließend genauer auf das industrielle Komplexitätsmanagement und die Problematik der Komplexitätsfalle ein. Diese beiden Stränge werden dann zusammengeführt, indem wir zeigen wir, wie Übertragung von Maßnahmen der Komplexitätsreduktion auf ITDienstleistungen aussehen kann. Dies wird anhand eines Fallbeispiels illustriert. Abschließend fassen wir die Nutzenpotenziale für Anbieter und Nachfrager dieser bedarfsorientierten IT-Outsourcingangebote zusammen und geben einen Ausblick auf weitere Forschung in diesem Bereich. 


\section{IT-Dienstleistungen aus Anbietersicht}

Dienstleistungen sind Leistungen, deren Ergebnisse bestimmte materielle oder immaterielle Wirkungen an den externen Faktoren darstellen, in deren Erstellungsprozesse externe Faktoren integriert werden, an denen oder mit denen die Leistung erbracht wird, und bei denen ein Leistungspotenzial existiert, welches die Fähigkeit und Bereitschaft zur Erbringung einer Leistung bereithält. [Klein01, S. 30; MeBr00, S. 30]. Dabei wird es als ausreichend angesehen, wenn diese Merkmale überwiegend vorliegen [Cors97, S. 30; MeBr00, S. 30].

IT-Dienstleistungen können durch den Objektbezug entlang der drei vorgenannten Dimensionen Leistungsergebnis, Leistungsprozess und Leistungspotenzial konstitutiv abgegrenzt werden. Das Leistungsergebnis zielt auf die Planung, Entwicklung, Bereitstellung, Unterstützung und/oder das Management von IT-Systemen oder durch IT-Systeme ermöglichte Geschäftsaktivitäten (in Anlehnung an [oVer01, S. 6]). IT-Systeme sollen verstanden werden als der informationstechnische Teil betrieblicher Informationssysteme. Dabei sind IT-Systeme oder auf sie bezogene Faktoren sowohl Gegenstand des Leistungspotenzials als auch externe Faktoren dieser Dienstleistungen [Böhm04, S. 32]. Institutionell gesehen werden IT-Dienstleistungen einerseits von selbständigen Unternehmen und andererseits unternehmensintern durch die Organisationseinheiten der Informationsverarbeitung erbracht.

Im Folgenden sollen die Leistungs- und Gestaltungselemente von IT-Dienstleistungen aus Anbietersicht herausgearbeitet werden, um die Komplexität von ITDienstleistungen untersuchen zu können.

Ein wesentliches Merkmal von IT-Dienstleistungen ist, dass sich im Leistungsergebnis, im Leistungserstellungsprozess und im Leistungspotenzial technische und organisatorische Gestaltungselemente verbinden (vgl. Abbildung 1). Dies soll zunächst am Beispiel der Bereitstellung eines betriebswirtschaftlichen Anwendungssystems mit vereinbarten Servicegraden verdeutlicht werden. Die vereinbarten Leistungen sollen hier die Funktionen des Systems, d. h. seine Performanz (z. B. Antwortzeiten) sowie seine Zuverlässigkeit (z. B. durchschnittliche Verfügbarkeit), umfassen. Die Erstellung einer solchen Leistung erfordert einerseits die Bereitstellung einer geeigneten Konfiguration von Systemelementen und andererseits die Durchführung von Serviceprozessen, durch die ein anforderungsgerechter Betrieb sichergestellt wird. Diese Serviceprozesse sorgen beispielsweise für die Migration von einem bestehenden System auf das neue, durch die Dienstleistung bereitgestellte Anwendungssystem, den sicheren Betrieb oder das regelmäßige Umsetzen erforderlicher Änderungen an den fachlichen Funktionen (Wartung).

Gestaltungselemente sind demnach zunächst IT-Systeme, an denen Leistungen erbracht oder die bereitgestellt werden. Dazu zählen Anwendungen mit ihren Funktionen und Daten sowie die für ihren Einsatz erforderlichen IKTInfrastrukturen [Krcm03, S. 47]. Für die Leistungserbringung oder Bereitstellung 
können Transformationen der IT-Systeme erforderlich sein, die durch Serviceprozesse bewirkt werden. Durch die Transformation ergeben sich gegenseitige Abhängigkeiten zwischen IT-Systemen und Serviceprozessen. Um die zugesicherten Leistungen zu erreichen, sind Systemelemente und Serviceprozessaktivitäten (begrenzt) füreinander substituierbar.

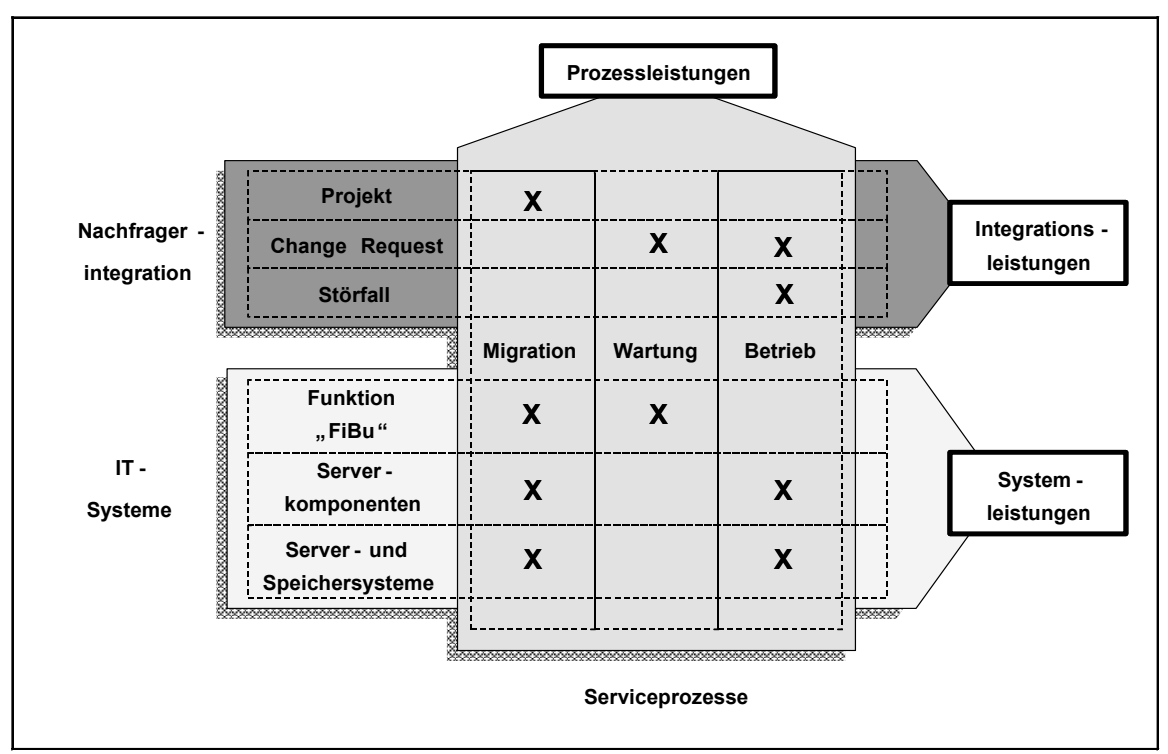

Abbildung 1: Leistungselemente von IT-Dienstleistungen (Vereinfachtes Beispiel aus [BöKr04])

Allerdings kann sich eine Beschreibung von IT-Dienstleistungen nicht auf ITSysteme und Serviceprozesse als zentrale Leistungselemente beschränken. Für Dienstleistungsanbieter ist die effektive und effiziente Integration von Nachfragern eine zentrale Kompetenz. Die Nachfragerintegration bedeutet bei IT-Dienstleistungen sowohl, dass externe IT-Systeme der Nachfrager genutzt oder verändert werden als auch, dass Mitglieder der Nachfragerorganisation in die Durchführung der Serviceprozesse eingebunden sind [Böhm04, S. 83ff.].

Insbesondere die Mitarbeiterintegration führt zu einer Sichtbarkeit von Leistungserstellungsprozessen [Shos84], die die wahrgenommene Qualität der Dienstleistung beeinflusst und den Nachfragern die Möglichkeit gibt, den Prozess mit zu steuern. Gleichzeitig definiert diese Schnittstelle zum Nachfrager auch die umgekehrte Verzahnung, d. h. wie sich die Serviceprozesse des Anbieters in die Aktivitäten der Nachfragerunternehmen einfügen. Daher besitzt für IT-Dienstleistungen die Nachfragerintegration und Gestaltung der Kundenbeziehung eine hohe Bedeutung [KeWi00]. Um diese gegenseitige Integration zu optimieren, nutzen Anbieter oftmals spezielle Instrumente. Ein Beispiel dafür ist das Service-Management, das die Zusammenarbeit von Mitarbeitern, Anbietern und Nachfragern überwacht und 
steuert. Neben IT-Systemen und Serviceprozessen kann daher auch die Gestaltung der Nachfragerintegration zur Spezifikation einer IT-Dienstleistung gezählt werden.

Diese Gestaltungselemente spiegeln sich auch in der Leistungssicht. Wegen der oft immateriellen Leistungsergebnisse von Dienstleistungen kommt ihrer vertraglichen Spezifikation gerade bei IT-Dienstleistungen eine besondere Bedeutung zu.

Durch die zumeist immateriellen Leistungsergebnisse von Dienstleistungen kommt der Definition der Leistungen eine besondere Bedeutung zu [Burr02; KeWi00, S. 12]. Für IT-Dienstleistungen sind sowohl die Vertragswerke als auch mögliche Methoden zur Messung der Leistungsqualität relativ weit entwickelt [Stu $\left.{ }^{+} 00\right]$. Diese steht jedoch in enger Verbindung mit den Leistungselementen der IT-Dienstleistungen, für die sie Vorgaben spezifiziert, die in der Gestaltung umgesetzt werden müssen und aus denen sich folglich auch Abhängigkeiten zwischen den Gestaltungselementen ergeben. Bezugsobjekte der Leistungen dann können IT-Systeme (z. B. Funktionen, Verfügbarkeiten, Antwortzeiten), Serviceprozesse (z. B. Aktivitäten, Reaktionszeiten, Betreuungszeiten) wie auch die Nachfragerintegration (z. B. Integrationsformen, Mitwirkungspflichten, Eskalation) sein. Dementsprechend lässt sich die Leistungssicht durch System-, Prozess- und Integrationsleistungen beschreiben.

Entlang dieser Leistungs- und Gestaltungselemente sollen in den späteren Abschnitten die Anwendungsmöglichkeiten für Maßnahmen des industriellen Komplexitätsmanagements auf IT-Dienstleistungen näher untersucht werden. Zuvor verdeutlicht der nächste Abschnitt aber das Problem der Komplexitätsfalle in der Industrie und zeigt, welche Maßnahmen zur Reduktion von Komplexität ergriffen werden können.

\section{Komplexitätsfalle und Komplexitätsmanagement}

Unter Komplexität wird „... das Zusammentreffen einer strukturellen Vielschichtigkeit, resultierend aus der Anzahl und Diversität der Elemente eines Systems sowie deren gegenseitige Verknüpfung und der dynamischen Veränderlichkeit der gegenseitigen Beziehungen der Systemelemente" verstanden [Pill00, S. 179] (nach [Blis00, S. 127]). Komplexitätskosten sind demnach Faktorverbräuche, die aus der Komplexität des Leistungsprogramms, der Kundenstruktur, der Produkte sowie dem Programm und der Organisation der Leistungserstellung resultieren [AdRo95; Meff00, S. 1043].

Die Komplexitätsfalle wird ausgelöst durch Differenzierungs- oder Individualisierungsdruck [AdJo98, S. 5]. Um Kunden zu gewinnen bzw. zu halten, werden individuelle Lösungen konzipiert und immer stärker differenzierte Leistungen angeboten. Pro Variante sinken die abgesetzten Volumina [AdJo98, S. 8]. 
Dem stehen jedoch steigende Komplexitätskosten gegenüber (vgl. zu den folgenden Ausführungen [AdJo98, S. 8f.; Pill00, S. 181]). Im Vertrieb führt die Differenzierung und Individualisierung zu höheren Ressourcenbedarfen für die Bearbeitung heterogener Märkte und für die kundenspezifische Angebotserstellung. In der Fertigung wächst z. B. die Anzahl der verwendeten Komponenten oder die Zahl der Aufträge mit geringen Wiederholeffekten und Losbildungsmöglichkeiten. Sowohl für den Fremdbezug als auch für die Eigenerstellung steigt der Ressourcenbedarf durch eine aufwändigere Koordination, Beschaffungslogistik oder Leistungserstellung. Diese höheren Ressourcenbedarfe führen zu steigenden Kosten. Auch Systeme zur Komplexitätsbeherrschung wie z. B. CIM-Systeme haben diese Kosten nicht nachhaltig senken können und tragen so paradoxerweise durch die erforderlichen Investitionen zu Kostensteigerungen bei [AdRo95].

Wenn keine verursachungsgerechte Verrechnung der Komplexitätskosten erfolgt, besteht die Gefahr der Quersubventionierung. Hochvolumige Standardprodukte werden zu teuer, Variantenprodukte mit geringer Stückzahl zu günstig angeboten. Treten nun im Bereich der Standardprodukte (commodity) Wettbewerber auf, so sind die Unternehmen in diesem Markt nicht mehr wettbewerbsfähig. Wegen der Quersubventionierung sind dadurch aber auch die Nischenmärkte bedroht [AdJo98, S. 14-22; Meff00, S. 1037-1039].

Welches Maß an Komplexität ist aber nun ,gut“ für ein Unternehmen? Diese Frage führt in der im Folgenden zusammengefassten Argumentation von Bliss [Blis00, S. 133-163] zu einem Trade-Off: Auf der einen Seite steht das statische Potenzial, mit dem ein Unternehmen auf Veränderungen der Umwelt reagieren kann. Je größer die Menge der Alternativen, mit denen Umweltveränderungen kompensiert werden können und so das Unternehmen als System stabilisiert wird, desto größer ist dessen potenzieller Fit mit zukünftigen Umweltzuständen und daher dessen Überlebensfähigkeit, desto höher aber auch die Komplexität.

Bei dieser besseren Umweltentsprechung durch ein Mehr an Alternativen besteht aber ein Trade-Off zum dynamischen Potenzial eines Unternehmens. Eine hohe Zahl von Elementen und möglicher Relationen zwischen diesen führt zu einer Selektionslast: Die Umweltveränderung erfordert, dass die zu ihr passenden Elemente und Relationen ausgewählt werden. Umgekehrt formuliert: Bei einer großen Zahl von Kombinationsmöglichkeiten muss ein großer Teil dieser Möglichkeiten ausgeschlossen werden. Reicht die Zeittoleranz für eine Reaktion auf eine Umweltveränderung nicht aus, um diesen Selektionsprozess durchzuführen, ist die Überlebensfähigkeit des Systems gefährdet. Aus der dynamischen Sicht wird daher eine möglichst geringe Komplexität des Systems nahe gelegt, damit ein Unternehmen nicht nur potentiell eine Umweltentsprechung erreichen kann, sondern sie unter den gegebenen (Zeit-)Restriktionen einer konkreten Situation auch erreicht.

Nicht alle Komplexitätstreiber wirken gleichsam auf beide Seiten. Die erforderliche Varietät zur Umweltentsprechung steht in seiner Sicht nur mit der Komplexität der Kundenstruktur, des Leistungsprogramms und der Produkte in Beziehung. 
Bliss bezeichnet sie als korrelierte Unternehmenskomplexität, weil sie sich direkt aus der wahrgenommenen Marktkomplexität ableitet. Dabei wird immer ein positiver Zusammenhang zwischen diesen Größen bestehen, jedoch kann das Unternehmen die Stärke des Zusammenhangs durch Gestaltungsmaßnahmen beeinflussen. Dagegen stehen für Bliss die Komplexität des Produktionsprogramms, der Organisation, der Ziele und des Fertigungssystems in keinem direkten Bezug zur wahrgenommenen Marktkomplexität. Diese autonome Komplexität kann daher ohne direkte Auswirkungen auf die Marktaufgabe verändert werden.

\begin{tabular}{|c|c|c|}
\hline $\begin{array}{l}\text { Reduktion der } \\
\text { autonomen } \\
\text { Unternehmenskomplexität }\end{array}$ & $\begin{array}{l}\text { Reduktion der } \\
\text { korrelierten } \\
\text { Unternehmenskomplexität }\end{array}$ & $\begin{array}{l}\text { Reduktion der wahrge- } \\
\text { nommenen Marktkomplexi- } \\
\text { tät }\end{array}$ \\
\hline $\begin{array}{ll}- & \text { Standardisierung } \\
\text { (Gleichteile- } \\
\text { verwendung, Funktions- } \\
\text { integration) } \\
\text { - } \quad \text { Horizontale Prozess- } \\
\text { integration } \\
\text { - Vertikale Autonomie }\end{array}$ & \begin{tabular}{|l|} 
Bündelung (Paketierung, \\
Premium-Standards, \\
Pflichtkonfiguration) \\
Modularisierung \\
- Verschiebung des Vari- \\
antenbestimmungs- \\
punktes
\end{tabular} & $\begin{array}{ll}- & \text { Kundenbereinigung } \\
\text { - } & \text { Programmbereinigung }\end{array}$ \\
\hline
\end{tabular}

Tabelle 1. Ausgewählte Maßnahmen zur Komplexitätsreduktion (Quelle: eigene Darstellung nach [Blis00, S. 197-204])

Aus diesen Zusammenhängen leitet Bliss einen Ordnungsrahmen für das Komplexitätsmanagement ab [Blis00, S. 170-215]. In einem ersten Schritt sollen demnach Maßnahmen des Komplexitätsmanagements darauf gerichtet sein, die autonome Komplexität eines Unternehmens zu reduzieren (vgl. Tabelle 1). Da diese keine direkten Auswirkungen auf die absatzmarktbezogene Varietät hat, wird damit die Fähigkeit des Unternehmens nicht verringert, auf vielfältige Anforderungen des Markts zu reagieren. Im nächsten Schritt gilt es, Maßnahmen zu ergreifen, die zu einem schwächeren Zusammenhang zwischen der wahrgenommenen Marktkomplexität und der korrelierten Komplexität führen. Erst danach sind aus seiner Sicht Maßnahmen sinnvoll, die die Anforderungen des Absatzmarktes so selektieren, dass dadurch eine geringere wahrgenommene Marktkomplexität und somit auch eine geringere korrelierte Unternehmenskomplexität entsteht. Als letzten Schritt sieht er Maßnahmen der Komplexitätsbeherrschung an, auf die aber in diesem $\mathrm{Zu}$ sammenhang nicht weiter eingegangen wird. In diesen Ordnungsrahmen ordnet Bliss nun verschiedene Maßnahmen des Komplexitätsmanagements ein. 


\section{Maßnahmen der Komplexitätsreduktion bei IT- Dienstleistungen}

Wie können diese Maßnahmen der Komplexitätsreduktion nun auf variantenreiche IT-Dienstleistungen angewendet werden? Der Ordnungsrahmen von Bliss [Blis00, S. 197-202] fordert als erste Maßnahme die Reduzierung der autonomen Komplexität, indem eine „Übererfüllung“ der erforderlichen Varietät abgebaut wird. In den folgenden Maßnahmen soll dann die Umsetzung marktbezogener Varietät in die korrelierte Unternehmenskomplexität verbessert werden. Dazu dienen die Verschiebung der Entkopplungspunkte, die Modularisierung sowie die Bündelung. Schließlich kann über eine geringere wahrgenommene Marktkomplexität die Komplexität weiter reduziert werden. Die Überlegungen sollen anhand eines Fallbeispiels erläutert werden, das im Folgenden kurz eingeführt wird.

\subsection{Das Fallbeispiel ALPHA}

ALPHA ist einer der führenden deutschen Anbieter von IT-Dienstleistungen für den Betrieb von Unternehmenssoftware mit über 500 Mitarbeitern und einem Jahresumsatz von über $€ 100$ Mio. Ein zentrales Leistungsangebot ist die Bereitstellung von individuell aufgebauten Anwendungssystemen (Application Hosting). Die Nachfrager greifen über Netzwerkverbindungen auf diese Systeme zu, so dass ALPHA den Betrieb der Server im eigenen Rechenzentrum konzentrieren kann. Die Leistungen sind vor allem auf die Bereitstellung bestimmter Standardsysteme (Produkte) ausgerichtet.

Der größte Teil der Abnehmer von Application-Hosting-Leistungen überträgt ALPHA die Verantwortung für den Betrieb von SAP-Systemen. Derzeit betreibt ALPHA über 300 SAP-Systeme für über 100 Kunden. Der geografische Schwerpunkt der unternehmerischen Aktivitäten liegt auf Deutschland und weiteren europäischen Ländern. $\mathrm{Zu}$ den Kernleistungen gehören die Implementierung oder Migration von SAP-Systemen, die Systembereitstellung in einer individuellen ITUmgebung, der Support für die Anwendung, die Wartung und Administration, sowie die Abstimmung und Verbesserung der Leistungen (vgl. [Böhm04, S. 116] für eine Übersicht), für die jeweils zahlreiche Anpassungsmöglichkeiten bestehen.

Im Rahmen der Diagnosephase eines Action-Research-Projektes [Bask99] wurde im Jahr 2001 zunächst eine Fallstudie durchgeführt, die den der Aufbau, die zentralen Abhängigkeiten sowie die interne und externe Beurteilung dieser ITDienstleistung durch 14 Interviews sowie Dokumenten- und Systemanalysen erhoben hat (ausführlicher dargestellt in [Böhm04, S. 105ff.]). Diese Erhebung wurde ergänzt durch eine Reihe von Workshops zur Modularisierung und Konfigurationsunterstützung von IT-Dienstleistungen in den Jahren 2002-2004, in denen Informationen über die Weiterentwicklung des Leistungsprogramm und der Organisation der Leistungserstellung gewonnen wurden. 
Dieses Leistungsangebot von ALPHA soll herangezogen werden, um darzustellen, wie einzelne Maßnahmen des industriellen Komplexitätsmanagements explizit oder implizit bereits umgesetzt wurden. Auf der einen Seite ist ein zentrales Leistungsversprechen, dass die IT-Umgebung auf die individuellen Anforderungen der Nachfrager ausgerichtet wird (Firmenbroschüre). Auf der anderen Seite ist das Leistungsangebot im Gegensatz zum klassischen Outsourcing auf vielfache Implementierung ausgelegt. Dadurch lassen sich in den Leistungs- und Gestaltungselementen eine Reihe von Umsetzungsbeispiele für Maßnahmen des Komplexitätsmanagements finden, da ALPHA für die erfolgreiche wiederkehrende Erbringung bereits die externe und interne Komplexität dieses Leistungsangebots reduzieren musste.

\subsection{Standardisierung und Prozessorientierung}

Die Standardisierung zielt auf den Abbau autonomer Komplexität. Dadurch wird die Varietät der Potenzialfaktoren und Prozesse des Unternehmens reduziert, ohne die am Markt angebotenen Leistungen einzuschränken.

Unter Standardisierung wird im verarbeitenden Gewerbe der verstärkte Einsatz von Normteilen zur Verringerung der Zahl funktional identischer Teile verstanden [Blis00, S. 42]. Damit können der Teilestamm reduziert und möglicherweise Skaleneffekte in der Produktion bzw. dem Fremdbezug dieser Teile erreicht werden.

Auch bei IT-Dienstleistungen kann autonome Komplexität abgebaut werden, indem IT-Systeme, Serviceprozesse und Formen der Nachfragerintegration auf funktional identische Elemente mit unterschiedlichen Implementierungen untersucht werden. Gerade durch Standardisierung können Skaleneffekte erzielt werden.

ALPHA versucht, einen möglichst hohen Teil der Kundenanforderungen durch Einsatz einer Baureihe von Servern zu erfüllen. Damit sollen zunächst günstigere Beschaffungskonditionen realisiert werden. Gleichzeitig können aber solche Standardkomponenten auch Teil eines verbesserten Ressourcenmanagements sein, bei denen frei werdende Komponenten einer weiteren Verwendung zugeführt werden. Vor der Verwendung einer anderen Reihe wird daher geprüft, ob nicht auch eine alternative Konfiguration der Standard-Baureihe die erforderlichen Leistungsdaten aufweist.

Die Standardisierung von IT-Systemen kann zudem zur Grundlage des Reengineering von Serviceprozessaktivitäten gemacht werden, die für die Erstellung von ITDienstleistungen an standardisierten IT-Systemelementen ausgeführt werden. Ein Beispiel bei ALPHA dafür ist, wenn Installation und Wartung solcher Standardkomponenten nach stark routinisierten Arbeitsplänen durchgeführt werden, um damit die Disposition von Serviceaufträgen zu vereinfachen und deren Erstellungskosten zu senken. 
Bei Serviceprozessaktivitäten sind die IT-Systeme jedoch nur ein Vielfaltstreiber. Der zweite, wichtige Treiber ist die Nachfragerintegration. Eine Standardisierung der Serviceprozessaktivitäten ist demnach nur dann möglich, wenn entweder nicht oder nur in geringem Maße Nachfrager in die Serviceprozesse integriert werden oder neben der Produktion mit standardisierten IT-Systemelementen auch noch die Integration der Nachfrager einheitlich gestaltet werden kann.

ALPHA hat für zentrale Leistungsangebote einheitliche Formen der Nachfragerintegration etabliert. Abhängig von der Phase der Nachfrager-Anbieter-Beziehung sind dazu bestimmte Rollen und Kommunikationsformen vorgesehen, so dass die Zusammenarbeit immer einem ähnlichen Muster folgt. Durch diese Standardisierung wird für alle Nachfrager die gleiche Linie der Sichtbarkeit bzw. der Mitwirkung an Serviceprozessen erreicht. Dies grenzt einheitlich integrative von nichtintegrativen Teilen ab und schafft somit auch Standardisierungsmöglichkeiten bei Serviceprozessaktivitäten und - in der Folge - auch bei IT-Systemen.

Die Nachfragerintegration ist neben der Standardisierung auch bei der Veränderung des Variantenbestimmungspunktes eine zentrale Einflussgröße.

\subsection{Verschiebung der Entkopplungspunkte}

Durch die Maßnahmen der Verschiebung der Entkopplungspunkte, der Modularisierung und der Bündelung soll die Übersetzung von wahrgenommener Marktkomplexität in korrelierte Unternehmenskomplexität verbessert werden.

Der Entkopplungspunkt oder Variantenbestimmungspunkt bezeichnet die Stelle im Fertigungsprozess, an der die auftragsneutrale, standardisierte Fertigung in eine auftragsspezifische Fertigung übergeht [Blis00, S. 46-47; Pill00, S. 232]. Dabei wird davon ausgegangen, dass die auftragsneutrale Vorfertigung durch Prozessstandardisierung mit einer geringeren Komplexität für die Planung und Steuerung der Produktion einhergeht. Die Fertigungsaufträge für die auftragsbezogene Fertigung werden dabei ausschließlich aufgrund von konkreten Kundenaufträgen ausgelöst, die Fertigungsaufträge für die auftragsneutrale Fertigung werden dagegen unabhängig von den konkreten Aufträgen geplant [Pill00, S. 232]. Wegen der geringeren Komplexität der auftragsneutralen Fertigung ist es das Ziel, den Entkopplungspunkt auf eine möglichst späte Wertschöpfungsstufe zu verlagern. Dadurch soll im auftragsneutralen Teil der Fertigung die Anzahl der zu berücksichtigenden Varianten gesenkt werden. Dies ermöglicht eine größere Standardisierung der Leistungserstellungsprozesse. Dazu sollen die Freiheitsgrade in der Planung und Steuerung der Leistungserstellung erhöht werden. Damit ist eine auftragsneutrale Disposition gemeint, die Effizienzvorteile in der Leistungserstellung, z. B. durch Losbildung, ausschöpfen kann [Pill00, S. 232-233]. 
Eine Anwendung auf IT-Dienstleistungen erfordert aber eine weitergehende Differenzierung in einen leistungsbezogenen und einen integrationsbezogenen Entkopplungspunkt.

Der leistungsbezogene Entkopplungspunkt folgt der Denkweise der industriellen Fertigung und zeigt auf, welcher Teil der Leistungserstellung auftragsneutral erfolgt und welcher Teil auftragsbezogen durchgeführt wird. Insgesamt erfolgt bei ALPHA nur in geringem Maß eine auftragsneutrale Leistungserstellung. Beispielsweise ist die Herstellung und Lagerung vorkonfigurierter Server oder anderer Systemkomponenten bei ALPHA nicht zu beobachten. Eine solche auftragsneutrale Leistungserstellung wäre allerdings auch nur dann sinnvoll, wenn die Effizienzvorteile der auftragsneutralen Fertigung die Kosten der Lagerhaltung und die Risiken einer auftragsneutralen Disposition überkompensierten. Letztere sind für die IT insofern oftmals sehr hoch, dass durch den technischen Wandel mit der Lagerung ein hoher Wertverlust für die IT-Vermögensgegenstände einhergeht. Das Bestandsrisiko ist noch erhöht, wenn zudem eine hohe Prognoseunsicherheit hinsichtlich der erforderlichen Ressourcen vorliegt. Dazu kommt, dass nur ein geringer Teil der Leistungen überhaupt lagerfähig ist, da bei vielen ITDienstleistungen Produktion und Konsum zusammenfallen (uno-actu-Prinzip). Auch hier ist eine auftragsneutrale Disposition nicht möglich. Daher findet die Vorfertigung in dem nachfragerunabhängigen Leistungspotenzial seine Entsprechung, dass Anbieter zur Gewährleistung der Leistungsbereitschaft vorhalten müssen [Klein01].

Eine Vorfertigung findet sich daher bei ALPHA nur bei technischen Basisdiensten. Unabhängig von konkreten Aufträgen werden dafür gemeinsam genutzte Systeme bereitgestellt und betrieben. Im Fall der Dienstleistungen von ALPHA finden sich Beispiele bei zentralen IT-Komponenten, wie z. B. für Rechenzentrumsräume, Netzwerk, Storage oder Datensicherung. Bereitstellung und Betrieb dieser Elemente wird auftragsneutral gewährleistet. Durch Bündelung der Anfragen aus den Kundenprojekten in zwei Bereichen können so Skaleneffekte oder Flexibilitätsvorteile eröffnet werden. Die so bereitgestellten Dienste werden dann in nachgelagerten Serviceprozessen noch auftragsspezifisch konfiguriert und dienen dann als Grundlage für die Realisierung von auftragsspezifischen Systemumgebungen.

Während der leistungsbezogene Entkopplungspunkt den Übergang von standardisierter zu auftragsspezifischer Leistungserstellung beschreibt, markiert der integrationsbezogene Entkopplungspunkt den Einstieg in die standardisierte Leistungserstellung.

Bei IT-Dienstleistungen ist vielfach eine auftragsneutrale Gestaltung und Disposition der Prozesse stark eingeschränkt. Bei einer umfassenden Integration externer Faktoren ist die Vielfalt in der Leistungserstellung nur sehr begrenzt planbar. Dies ist beispielsweise der Fall, wenn der Anbieter im Rahmen der Dienstleistung ITSysteme der Nachfrager betreibt oder wartet. Genauso kann bei Projektarbeiten eine umfangreiche Integration von Mitarbeitern der Nachfrager erforderlich sein. 
Eine auftrags- und nachfragerunabhängige Leistungserstellung ist dann für die Wertschöpfungsstufen, in denen diese integrationsbezogene Vielfalt vorherrscht, weitgehend ausgeschlossen. Der Punkt in der Wertschöpfungskette, bis zu dem externe Faktoren überwiegend unverändert integriert werden müssen, soll daher als integrationsbezogener Entkopplungspunkt bezeichnet werden. Erst ab diesem Punkt können zumindest Teile der Leistungserstellung unabhängig von auftragsspezifischen externen Faktoren gestaltet werden.

Um den integrationsbezogenen Entkopplungspunkt möglichst weit an den Anfang der Wertschöpfungskette zu verlegen, ist entsprechend eine Veränderung der Integration externer Faktoren erforderlich. Dazu müssen externe IT-Systeme sowie die Formen der Nachfragerintegration angepasst werden. Diese Anpassung ist ein Kernelement der Application-Hosting-Dienstleistung der ALPHA.

Durch eine Migration bestehender Systeme wird der externe Faktor auf die nachfragerspezifische Anpassung des SAP-R/3-Systems reduziert. Dabei werden ausschließlich das Customizing, die Erweiterungen und Modifikationen von Programmen, die Benutzerstruktur sowie die Anwendungsdaten des Systems übernommen. Das wird in SAP-R/3 dadurch ermöglicht, dass alle diese Teile durch das Basismodul von der darunter liegenden IT-Plattform sowie vom konkreten Datenbankmanagementsystem entkoppelt werden. Diese Anpassungen werden im Zuge der Migration durchgeführt, die für eine Verlagerung eines bestehenden Systems in das Rechenzentrum der ALPHA erforderlich ist.

Die Migration ist erforderlich, um die Systeme in das Rechenzentrum der ALPHA zu übernehmen. Dabei erfolgt aber zugleich eine Anpassung der IT-Plattform und des Datenbankmanagementsystems. Dadurch reduziert sich für ALPHA die Varietät der externen Faktoren auf eine kleine Anzahl von bestimmbaren Systemkomponenten. Als Standard stellt ALPHA den Nachfragern zwei Typen von ITPlattformen sowie drei Datenbankmanagementsysteme zur Auswahl, während für SAP-R/3 prinzipiell eine viel höhere Zahl von Systemplattformen und Datenbankmanagementsystemen eingesetzt werden kann.

In einem gewissen Umfang liegt bei den Nachfragern eine Zahlungsbereitschaft für derartige Migrationsleistungen vor. Dies gilt vor allem dann, wenn die wirtschaftliche Nutzungsdauer der Plattform bzw. des Datenbankmanagementsystems erreicht ist oder strategische Gründe für einen Wechsel von wenig verwendeten zu weit verbreiteten Basissystemen sprechen. Ein weiterer Grund kann eine geplante, größere Softwarewartung sein, bei dem umfangreichere Änderungen am System vorgenommen werden müssen. Alle diese Fälle können durch eine solche Migration abgedeckt, den Nachfragern direkten Nutzen stiften und damit eine Zahlungsbereitschaft auslösen. Dies gilt vor allem dann, wenn durch die Migration kein technischer lock in erzeugt wird. ALPHA migriert daher die Systeme auf weit verbreitete Plattformen und Datenbankmanagementsysteme.

Gekoppelt ist diese technische Reduzierung der Nachfragerintegration mit einer organisatorischen. Während in der Implementierungs- und Migrationsphase eine 
intensive und nachfragerspezifsche Integration erfolgt, wird diese nach Inbetriebnahme der Systeme bei ALPHA vereinheitlicht und entkoppelt. In der Implementierungs- und Migrationsphase wird eine Projektorganisation eingerichtet, die eine enge Interaktion zwischen den Mitarbeitern von ALPHA und denen des Nachfragers umfasst. In der Betriebsphase verändert sich die Interaktion zwischen ALPHA und den Nachfragern deutlich. Im Wesentlichen erfolgt die Interaktion nun zwischen benannten Ansprechpartnern des Nachfragers und dem Call-Center von ALPHA, das eine zentrale Steuerung von Anfragen und Aufträgen vornimmt. Die Interaktion ist insofern stärker formalisiert, da nun vertraglich vereinbarte Reaktionszeiten wie auch Berichtsformen für die Leistungserfüllung gelten.

Durch diese technischen und organisatorischen Anpassungsmaßnahmen gelingt es ALPHA, den integrationsbezogenen Entkopplungspunkt an den Anfang der Leistungserstellung zu legen, um damit die Standardisierungspotenziale für spätere Wertschöpfungsstufen zu erhöhen. Insbesondere die Kapselung der Nachfragerintegration in der Betriebsphase sowie die weitgehende Ausschöpfung der Möglichkeiten durch technische Vereinheitlichung der Systeme geben ALPHA die Chance, bestimmte IT-Systemelemente und die damit verbundenen Serviceprozessaktivitäten autonom zu gestalten. Damit hat ALPHA höhere Freiheitsgrade bei der Bestimmung des leistungsbezogenen Entkopplungspunkts.

Durch die Verschiebung des integrationsbezogenen und leistungsbezogenen Entkopplungspunkts soll die Leistungserstellung bei IT-Dienstleistungen so gestaltet werden, dass die Komplexitätswirkungen der Integration externer Faktoren und der Erstellung variantenreicher Leistungen gedämpft werden. Dadurch ergeben sich Spielräume für eine umfassendere Standardisierung als auch für eine Modularisierung der IT-Dienstleistungen.

\subsection{Modularisierung}

Für variantenreiche Produkte liegt in der Produktarchitektur ein zentraler Ansatzpunkt zur Reduzierung der durch die Varietät am Markt induzierten internen Varietät des Anbieterunternehmens. Durch eine modulare Architektur kann vor allem die kombinatorische Flexibilität von Leistungsangeboten erhöht werden, ohne dass gleichzeitig die interne Komplexität von Entwicklung, Vertrieb und Produktion proportional ansteigt.

Eine modulare Architektur setzt eine Zerlegung des Produkts voraus [BaCl00, S. 64; Sanc96, S. 65; Ulri95, S. 423]. Eine solche Zerlegung wird als modular bezeichnet, wenn die Module untereinander lose gekoppelt sind $[\mathrm{BaCl00}$, S. 63; Sanc96, S. 65; Ulri95, S. 423]. Damit verbinden sich eine Reihe von Nutzenpotenzialen. Zunächst strukturieren sie Entwicklungsinformationen und Abläufe in der Produktentwicklung. Das wohl bedeutendste Potenzial modularer Produktarchitekturen liegt aber in der Eröffnung von Optionen für die Entwicklung und Konfiguration von Leistungsangeboten. Im Gegensatz zu integral aufgebauten 
Produkten erlauben modulare Servicearchitekturen, einzelne Module unabhängig zu verändern oder neu zu kombinieren. Ferner eröffnen sie Möglichkeiten zur Wiederverwendung von Modulen in unterschiedlichen Leistungsangeboten, zur unabhängigen Weiterentwicklung einzelner Module, zu ihrer selektiven Verwendung in nachfragerindividuellen Konfigurationen, zur Teilstandardisierung von Produkten sowie zur lokalen kontinuierlichen Verbesserung [BaCl00; SaMa96; Ulri95].

Allgemein gesprochen bieten modular aufgebaute Produkte und Dienstleistungen dort besondere Vorteile, wo sich Anbieter sowohl heterogenen Anforderungen der Kunden als auch heterogenen Inputfaktoren mit unterschiedlichen Lebenszyklen gegenüber sehen [Schi00]. Diese Überlegungen lassen sich generell auf Dienstleistungen [BöKr02; Burr02; $\mathrm{Hoo}^{+} 99$ ] und speziell auf IT-Dienstleistungen übertragen [Böhm04; BöKr04].

Auch bei IT-Dienstleistungen kann die Modularisierung zu entsprechenden Nutzenpotenzialen führen. ALPHA ist bestrebt, die Komplexitätserhöhung, die mit einer sukzessiven Ausweitung des Leistungsprogramms einhergeht, durch eine verstärkte Modularisierung der Dienstleistungen in Teilen zu kompensieren. Derzeit erfolgt die Modulbildung allerdings auf Ebene von Auftragstypen und grober Dienstleistungsphasen, so dass erst eine geringe Unterstützung für Entwicklung und Konfiguration daraus erwächst. Allerdings steht hier der Prozess erst am Anfang. Ein detaillierteres Anwendungsbeispiel findet sich in [Böhm04].

Durch die Modularisierung entwickeln Anbieter einen kombinierbaren Baukasten von Teildienstleistungen. Die Umsetzung heterogener Kundenanforderungen soll so auf die Kombination dieser Bausteine verlagert werden. Werden den Nachfragern aber sehr detaillierte Anpassungsmöglichkeiten angeboten, so kann diese Varietät im Detail oftmals noch nicht von der Modularisierung allein aufgefangen werden. Daher sollte im nächsten Schritt geprüft werden, ob die Anpassungsmöglichkeiten der Leistungen durch Bündelung und Verdichtung vereinfacht werden können.

\subsection{Bündelung}

Durch Bündelung bleibt die marktbezogene Varietät erhalten, wird aber intern verdichtet. Dies geschieht durch eine Zusammenfassung von Leistungsmerkmalen und Anpassungsmöglichkeiten. Dadurch können diese nur noch gemeinsam ausgewählt und nicht mehr einzeln angepasst werden. Dies kann auf unterschiedlichen Wegen erfolgen [Blis00, S. 40-41; Meff00, S. 1050]:

- Höherwertige Basisprodukte: Anstelle eines Basisprodukts, dass durch detaillierte Konfiguration erweitert werden kann, werden mehrere Varianten komplett ausgestatteter Basisprodukte angeboten. 
- Zwangskombinationen/Pakete: Bestimmte Leistungsmerkmale werden zu Zwangskombinationen bzw. Paketen zusammengefasst, die nur noch gesamthaft bezogen werden können. Dies kann auch eine Auslegung bestimmter Leistungsmerkmale auf die höchste Qualitätsstufe im Leistungsprogramm beinhalten. Zwar werden dadurch die Anforderungen bestimmter Nachfragergruppen übererfüllt, doch sollen die damit verbundenen Mehrkosten durch geringere Komplexitätskosten in Vertrieb und Leistungserstellung überkompensiert werden.

Diese Maßnahmen reduzieren die Komplexität in Vertrieb und Produktion. Der Vertrieb der entsprechenden Leistungsangebote wird vereinfacht, weil die nachfragerspezifische Anpassung entfällt oder weniger umfangreich wird. Die Produktion wird vereinfacht, weil ein geringerer Detaillierungsgrad bei der Produktionsplanung erforderlich ist. Insbesondere kann es dadurch gelingen, die Anpassung stärker auf Modulebene zu verlagern.

Zu diesem Zweck setzt auch ALPHA diese Maßnahmen ein. Damit soll Vertrieb und Controlling von Zusatzleistungen vereinfacht werden, die ALPHA auf Wunsch der Nachfrager im laufenden Betrieb erbringt. Zusatzleistungen sind wiederkehrende Prozessleistungen, über die Kunden beispielsweise einfache Konfigurationsänderungen in der Software, einzelne Wartungsarbeiten oder Administrationsaufgaben anfordern, die nicht primär zur Aufrechterhaltung eines zuverlässigen Betriebs der Anwendungssysteme erforderlich sind. Da diese Arbeiten von Nachfragern abgerufen werden können, hat ALPHA ein Interesse an einer Limitierung dieser Anforderungen, damit die Kosten für einzelne Nachfrager nicht unbegrenzt steigen können. Ursprünglich konnten für alle Leistungen dieser Art einzeln Mengenvarianten konfiguriert werden. Die Vereinbarung, Kalkulation und Kontrolle dieser einzelnen Limits hat sich aber als sehr aufwändig herausgestellt. Daher wurden diese Zusatzleistungen nun von ALPHA gebündelt und ihre Abrechnung über den Zeitverbrauch normiert. Für diese Leistungsbündel können die Kunden dann im Vertrag monatliche Zeitbudgets erwerben, auf die die ausgeführten Arbeiten dann angerechnet werden. In den Vertragsverhandlungen muss so nur ein einziger Wert vereinbart und konfiguriert werden, der zudem über Ressourcenverbräuche einfach zu kontrollieren ist.

Durch die Maßnahmen der Verschiebung der Entkopplungspunkte, der Modularisierung sowie der Bündelung kann das Verhältnis von marktbezogener Varietät zu interner Komplexität verbessert werden, ohne dass dadurch die marktbezogene Varietät eingeschränkt wird. Dort ist nun anzusetzen, wenn diese Maßnahmen noch nicht den erhofften Effekt bringen. Durch eine Programm- oder Kundenbereinigung wird die wahrgenommene Marktkomplexität reduziert. 


\subsection{Programm- und Kundenbereinigung}

Die Bereinigung des Leistungsprogramms und der Kundenstruktur hat zum Ziel, Leistungsangebote oder Kunden mit einem zu ihrer absoluten Anzahl stark unterproportionalen Umsatz- oder Produktionsanteil abzubauen, um die damit verbundene Komplexität in Auftragsabwicklung und Produktion zu reduzieren [Blis00, S. 39, 41].

Bei IT-Dienstleistungen ist ein solcher Ansatz zur Komplexitätsreduktion prinzipiell genauso anwendbar. Das Beispiel der ALPHA zeigt, dass dies vor allem im Hinblick auf die Reduzierung der Komplexität erforderlich ist, die durch die Integration externer Faktoren induziert wird.

Hinsichtlich der organisatorischen Nachfragerintegration fokussiert ALPHA interne IT-Abteilungen als Kunden. Die Leistungsangebote werden explizit als verlängerte Werkbank dieser Organisationseinheiten verstanden. Damit soll vor allem vermieden werden, dass ALPHA in die Abstimmung über die strategische Verwendung von IT einbezogen wird. Dies würde komplexe Kommunikationsbeziehungen zu einer Vielzahl von Fachabteilungen implizieren. Mit der Ausrichtung auf die interne IT als Kunden können die Integrationsformen auf die Schnittstelle zur IT-Organisation ausgerichtet werden. Die komplexen Kommunikationsbeziehungen, die für die Integration der unterschiedlichen fachlichen Anforderungen und Prioritäten an die IT zu berücksichtigen wären, entfallen.

Gleiches lässt sich für die technische Integration sagen. Hier kann die Vielfalt selbst bei der Integration von IT-Systemen, die auf Standardsoftware beruhen, stark steigen. Unterschiedliche Versionsstände, Modifikationen von Systemstandards sowie eine Vielzahl von Kopplungsmöglichkeiten mit anderen Systemen können für ALPHA zu einer hohen Heterogenität bei den externen Faktoren führen, wenn diese unverändert integriert werden. ALPHA homogenisiert diese Vielfalt durch eine Beschränkung möglicher externer Faktoren, womit implizit auch eine Selektion der Kunden erfolgt.

Die Leistungserstellung wird auf solche Systeme beschränkt, bei denen ein zentraler Betrieb der Systeme möglich ist. Die Architektur dieser Systeme muss dazu eine entsprechende Verteilung der Prozesse und Zugriffspunkte ermöglichen. Damit können einzelne IT-Systeme aus komplexeren Anwendungslandschaften der Kunden herausgelöst werden. ALPHAs wichtigste Leistungsangebote zielen hier auf bestimmte Anwendungssysteme wie z. B. SAP R/3. Damit wird die Heterogenität der externen Faktoren stark reduziert. Bei individuellen Lösungen erfolgt dies durch die Selektion von Kunden, deren Systeme auf Standardkomponenten aufsetzen, für die ALPHA die erforderlichen Serviceprozesse für Betrieb und Wartung bereits implementiert hat. Dazu zählen z. B. bestimmte Systemarchitekturen, Systemplattformen und Datenbankmanagementsysteme. Auch damit sollen die Anforderungen an die Integration externer Faktoren homogenisiert werden. 
Die mögliche Heterogenität bei der Integration externer Systeme kann ohne Auswirkungen auf die zentralen Serviceprozesse durch die Migration dieser Systeme ausgeweitet werden. Damit werden die externen Systeme so angepasst, dass Aufbau, Plattformen und Basiskomponenten (wie z. B. Datenbankmanagementsysteme) auf das Portfolio von ALPHA passen. Dies ist aber nur dort zu vertretbaren Kosten möglich, wo die Architektur der Systeme eine solche Flexibilität vorsieht.

\section{Fazit und Ausblick}

Die Entwicklung hin zu IT-Dienstleistungen, die auf Abruf verfügbar sind und nutzungsabhängig verrechnet werden, stellt Anbieter vor die Herausforderung, wie sie in der Folge der steigenden Komplexität der Leistungsangebote begegnen. Neue Technologien der Virtualisierung und Automatisierung von ITDienstleistungen erweitern die Möglichkeiten zur Komplexitätsbeherrschung. Das Beispiel der industriellen Produktion weist aber deutlich auf die Gefahren der Komplexitätsfalle hin, bei der eine zunehmende Differenzierung nicht zu höheren Erträgen, wohl aber zu deutlich höheren Kosten der Komplexitätsbeherrschung führt.

Anbieter von IT-Dienstleistungen sollten daher die Reduktion vor die Beherrschung der Komplexität stellen. Der Beitrag hat gezeigt, dass sich Maßnahmen aus dem industriellen Komplexitätsmanagement auf IT-Dienstleistungen adaptieren lassen. Das Fallbeispiel unterstreicht, dass derartige Maßnahmen in Einzelfällen bereits explizit oder implizit zur Anwendung kommen. Größere Nutzenpotenziale verspricht allerdings die systematische Ausschöpfung der Möglichkeiten entlang des vorgestellten Ordnungsrahmens.

Offen bleibt damit aber die Frage, wie Maßnahmen der Komplexitätsreduktion am besten mit erweiterten Möglichkeiten der Komplexitätsbeherrschung zusammenspielen. Wie und in welchem Umfang können z. B. die Potenziale der Virtualisierung von IT-Ressourcen durch eine Verschiebung von Entkopplungspunkten ausgeschöpft werden? Welche neuen Möglichkeiten für Standardisierung, Modularisierung und Bündelung ergeben sich durch Web-Services-Standards?

Die Erfahrungen des industriellen Komplexitätsmanagements legen nahe, dass erst die Kombination von Komplexitätsreduktion und -beherrschung hinreichend für die erfolgreiche Umsetzung von innovativen Dienstleistungsmodellen wie OnDemand-Services ist. Die hier vorgestellte Argumentation kann damit zum Ausgangspunkt für eine umfassendere Überprüfung von Erfolgsfaktoren dieser neuen Modelle aus Anbietersicht werden. 


\section{Literatur}

[AdJo98] Adam, D.; Johannville, U. (1998). Die Komplexitätsfalle. In: Adam, D. (Hrsg.), Komplexitätsmanagement, 5-28. Wiesbaden: Gabler.

[AdRo95] Adam, D.; Rollberg, R. (1995). Komplexitätskosten. Die Betriebswirtschaft, 55(5), 667-670.

[AnSt98] Ang, S.; Straub, D.W. (1998). Production and transaction economies and IS outsourcing: a study of the U.S. banking industry. MIS Quarterly(December), 535-552.

[BaCl00] Baldwin, C.Y.; Clark, K.B. (2000). Design Rules: The Power of Modularity (Bd. 1), Cambridge (MA), London: MIT Press.

[Bask99] Baskerville, R. (1999). Investigating Information Systems with Action Research. Communications of the Association of Information Systems, 2(19).

[Blis00] Bliss, C. (2000). Management von Komplexität: Ein integrierter, systemtheoretischer Ansatz zur Komplexitätsreduktion, Wiesbaden: Gabler.

[Böhm04] Böhmann, T. (2004). Modularisierung von IT-Dienstleistungen: Eine Methode für das Service Engineering, Wiesbaden: DUV.

[BöKr02] Böhmann, T.; Krcmar, H. (2002). Modulare Servicearchitekturen. In: Bullinger, H.-J.; Scheer, A.W. (Hrsg.), Service Engineering: Entwicklung und Gestaltung innovativer Dienstleistungen, 391-415. Heidelberg: Springer.

[BöKr04] Böhmann, T.; Krcmar, H. (2004). Grundlagen und Entwicklungstrends im ITServicemanagement. HMD - Praxis der Wirtschaftsinformatik(237), XX-YY.

[Burr02] Burr, W. (2002). Service Engineering bei technischen Dienstleistungen: eine ökonomische Analyse der Modularisierung, Leistungstiefengestaltung und Systembündelung (Bd. 286), Wiesbaden: DUV.

[Carr03] Carr, N.G. (2003). IT doesn't matter. Harvard Business Review, 81(5), 41-49.

[Cors97] Corsten, H. (1997). Dienstleistungsmanagement, 3 Aufl., München: Oldenbourg.

[Hoo $\left.{ }^{+} 99\right]$ Hoogeweegen, M.R.; Teunissen, W.J.M.; Vervest, P.H.M. (1999). Modular Network Design: Using information and communication technology to allocate production tasks in a virtual organization. Decision Sciences, 40(4), 1073-1104.

[KeWi00] Kern, T.; Willcocks, L.P. (2000). Exploring information technology outsourcing relationships: theory and practice. Journal of Strategic Information Systems, 9(4), 321350 .

[Klein01] Kleinaltenkamp, M. (2001). Begriffsabgrenzungen und Erscheinungsformen von Dienstleistungen. In: Bruhn, M.; Meffert, H. (Hrsg.), Handbuch Dienstleistungsmanagement, 27-50. Wiesbaden: Gabler.

[Krcm03] Krcmar, H. (2003). Informationsmanagement, 3 Aufl., Heidelberg: Springer.

[Lamm04] Lammers, M. (2004). Make,Buy or Share: Combining Resource Based View,Transaction Cost Economics and Production Economies to a Sourcing Framework. Wirtschaftsinformatik, 46(3), $204-212$. 
[LeRo03] Levina, N.; Ross, J.W. (2003). From the Vendor's Perspective: Exploring the Value Proposition in Information Technology Outsourcing. MIS Quarterly, 27(3), 331364.

[MeBr00] Meffert, H.; Bruhn, M. (2000). Dienstleistungsmanagement, 3 Aufl., Wiesbaden: Gabler.

[Meff00] Meffert, H. (2000). Marketing: Grundlagen marktorientierter Unternehmensführung, 9 Aufl., Wiesbaden: Gabler.

[oVer01] o.V. (2001). The Building Blocks of the Services Industry (Document 26051): IDC.

[Pill00] Piller, F.T. (2000). Mass Customization: Ein wettbewerbsstrategisches Konzept im Informationszeitalter, Wiesbaden: Gabler.

[SaMa96] Sanchez, R.; Mahoney, J.T. (1996). Modularity, flexibility, and knowledge management in product and organization design. Strategic Management Journal, 17(Winter Special Issue), 63-76.

[Sanc96] Sanchez, R. (1996). Strategic Product Creation: Managing New Interactions of Technology, Markets, and Organizations. European Management Journal, 14(2), 121138.

[Saun02] Saunders, C. (2002). Outsourcing: A View from Those Doing the Work. In: Hirschheim, R.; Heinzl, A.; Dibbern, J. (Hrsg.), Information Systems Outsourcing: Enduring Themes, Emergent Patterns and Future Directions, 329-347. Berlin, Heidelberg et al.: Springer.

[Schi00] Schilling, M.A. (2000). Toward a General Modular Systems Theory and Its Application to Interfirm Product Modularity. Academy of Management Review, 25(2), 312-333.

[Shos84] Shostack, G.L. (1984). Designing services that deliver. Harvard Business Review, 62(1), 133-139.

[Stu $\left.{ }^{+} 00\right]$ Sturm, R.; Morris, W.; Jander, M. (2000). Foundations of Service Level Management, Indianapolis, IN: Sams.

[Ulri95] Ulrich, K. (1995). The role of product architecture in the manufacturing firm. Research Policy, 24(3), 419-441. 